Check for updates

Cite this: RSC Adv., 2018, 8, 3803

\title{
Self-exfoliation of 2D covalent organic frameworks: morphology transformation induced by solvent polarity $\dagger$
}

\author{
Na Zhang, ${ }^{a}$ Taisheng Wang, ${ }^{a}$ Xing $\mathrm{Wu}{ }^{a}$ Chen Jiang, ${ }^{a}$ Fang Chen, ${ }^{a}$ Wei Bai ${ }^{\star b}$ \\ and Ruke Bai (D) *a
}

\begin{abstract}
Recently, covalent organic nanosheets (CONs) have emerged as functional two-dimensional (2D) materials for versatile applications. Strong interaction among layers and the instability of borate ester in moisture are the major hurdles to obtain few layered boron-containing CONs by exfoliation of their bulk counterparts. In this paper, we report a facile approach for preparation of few layered borate ester-containing CONs based on electrostatic repulsion of ions. We incorporated organic ionic groups into porous covalent organic frameworks (COFs) and it has been proved that the COFs with quaternary ammonium group could selfexfoliate into few layered ionic covalent organic nanosheets (iCONs) in polar organic solvents. Interestingly, the morphology of the iCOFs-A could be changed from a multilayered aggregation to nanocapsules, or 2D sheets when solvents with different polarity were used. In contrast, non-ionic covalent organic frameworks COFs-B could not self-exfoliate in various solvents. In addition, the selfexfoliated nanosheets could be used to fabricate uniform thin films on $\mathrm{SiO}_{2}$ wafer and the film exhibited explicit optical and electrical properties.
\end{abstract}

Received 30th August 2017
Accepted 9th January 2018

DOI: $10.1039 / \mathrm{c} 7 \mathrm{ra0} 0647 \mathrm{j}$

rsc.li/rsc-advances

delamination ${ }^{35}$ and chemically delamination. ${ }^{36}$ However, these exfoliation approaches unavoidably cause some defects in structure of the materials, which obviously affect their properties and limit their applications. Therefore, it is highly desired to develop a facile approach for preparation of $2 \mathrm{D}$ CONs in solution.

It is well known that the interaction of the polymer chains with inbuilt ionic character will be decreased due to electrostatic repulsion. On this account, it is possible to develop a selfexfoliating approach for preparation of 2D CONs. For example, self-exfoliation of imine-based COFs with positive charges was reported in water without external stimuli. ${ }^{37}$ However, it should be noticed that borate ester is not stable in moisture ${ }^{38}$ or in water, ${ }^{39}$ thus, exfoliation in water or aqueous solution is not feasible for the boron-based COFs. Actually, it is quite a challenge to achieve self-exfoliation of $2 \mathrm{D}$ boron-containing $\mathrm{COFs}$ in solution.

The COFs of boronate esters, the most investigated materials among the family of COFs, are prepared by condensation of boronic acids and catechols. However, it is well known that catechols are prone to oxidation and have a poor solubility in most organic solvents. We presumed that the in situ formation of catechols from protected catechols could prevent oxidation of catechols, and moreover, the protected catechols would possess higher solubility than the catechols in organic solvents. On the other hand, it is not easy to introduce ionic groups into the COFs of boronate esters because borate ester is not stable towards water or moisture. Fortunately, we found 
that $\mathrm{BF}_{3} \cdot \mathrm{OEt}_{2}$ as a Lewis acid catalyst could not only catalyze the formation of boron-containing COFs, ${ }^{\mathbf{4 0 , 4 1}}$ but also coordinate with nitrogen atoms to form organic ion pairs. Therefore, we successfully prepared the iCOFs of boronate esters by one-pot method using $\mathrm{BF}_{3} \cdot \mathrm{OEt}_{2}$ as catalyst. This one-pot process is a more convenient way for preparation of boronate iCOFs. The results demonstrated that few-layered ionic covalent organic nanosheets (iCONs) were obtained via two steps, formation of the iCOFs in low polar organic solvents and their self-exfoliation in high polar organic solvents. Moreover, the iCONs could be well dispersed in solution and a uniform thin film of the exfoliated iCONs was easily fabricated on $\mathrm{SiO}_{2}$ wafer, which exhibited explicit optical and electrical properties. To the best of our knowledge, this is the first report on the self-exfoliation of boroncontaining COFs in solution.

\section{Experimental}

\section{General}

All solvents were dried following the standard procedures before use. Unless otherwise indicated, all starting materials were obtained from commercial suppliers and used without further purification.

\section{Synthesis of compound 1}

A solution of 4-bromobenzaldehyde $(5.55 \mathrm{~g}, 30 \mathrm{mmol})$ and veratrole $(2.8 \mathrm{~g}, 20 \mathrm{mmol})$ in chloroform $(20 \mathrm{~mL})$ was added dropwise to a freshly prepared Eaton's reagent (7.7 wt\% phosphorus pentoxide solution in methanesulfonic acid) in an icebath. After addition, the suspension was stirred for $2 \mathrm{~h}$ under reflux and then cooled to room temperature. Then DDQ (1 equiv.) was added to the mixture and the reaction was heated at $40{ }^{\circ} \mathrm{C}$ for $2 \mathrm{~h}$. Subsequently, the organic mixture was poured into iced water and neutralized with $2 \mathrm{M} \mathrm{NaOH}$. After extraction with chloroform, the solvent was concentrated under reduced pressure and the residue was recrystallized from a methanol-chloroform mixture to give 1 with a yield of $25 \% .{ }^{1} \mathrm{H}$ NMR $(300 \mathrm{MHz}$, $\left.\mathrm{CDCl}_{3}\right): \delta 7.74(\mathrm{~d}, 4 \mathrm{H}), 7.37(\mathrm{~d}, 4 \mathrm{H}), 6.75(\mathrm{~s}, 4 \mathrm{H}), 3.76(\mathrm{~s}, 12 \mathrm{H}) .{ }^{13} \mathrm{C}$ NMR (100 MHz, $\left.\mathrm{CDCl}_{3}\right): \delta$ 149.2, 138.7, 132.8, 132.1, 131.9, 125.7, 121.8, 103.6, 55.7.

\section{Synthesis of compound 2}

Compound 1 (1.5 g, $2.47 \mathrm{mmol}$ ) was suspended in $47 \% \mathrm{HBr}(\mathrm{aq})$ $(200 \mathrm{~mL})$ and HOAc $(200 \mathrm{~mL})$ and the suspension was deoxygenated by passing a stream of Ar through the suspension for $5 \mathrm{~min}$. Then, the mixture was refluxed overnight under Ar. After $24 \mathrm{~h}$, the dark solution was allowed to cool slowly under Ar. The product as a crystalline solid was filtered, washed with water and dichloromethane in sequence. Since it is somewhat sensitive to air, the solid should be stored under Ar. Compound 2 was obtained in a yield of $83.3 \%(1.15 \mathrm{~g}) .{ }^{1} \mathrm{H}$ NMR $\left(300 \mathrm{MHz}, \mathrm{d}_{6}\right.$ DMSO): $\delta 7.80(\mathrm{~d}, 4 \mathrm{H}), 7.34(\mathrm{~d}, 4 \mathrm{H}), 6.62(\mathrm{~s}, 4 \mathrm{H}) .{ }^{13} \mathrm{C}$ NMR $(100$ MHz, $\mathrm{d}_{6}$-DMSO): $\delta 146.4,139.3,133.2,131.7,129.1,124.9,120.5$, 106.1.

\section{Synthesis of compound C2}

$1 \mathrm{~g}$ (1.8 mmol) of compound 2 was dissolved in $75 \mathrm{~mL}$ of benzene. To this solution was added $15 \mathrm{~mL}$ of acetone and $300 \mathrm{mg}$ of $p$-toluenesulfonic acid. The solution was heated to reflux overnight in a Dean-Stark apparatus. After the collection of water ceased, the reaction solution was diluted with $200 \mathrm{~mL}$ of $\mathrm{CH}_{2} \mathrm{Cl}_{2}$ and washed with water $(2 \times 100 \mathrm{~mL})$. The organic layers were dried over $\mathrm{Na}_{2} \mathrm{SO}_{4}$ and the solvent was removed by rotary evaporation. The residue was purified by column chromatography using silica gel with a mixture of petroleum ether and dichloromethane $(1: 1, \mathrm{v} / \mathrm{v})$ as eluent to give $\mathbf{C 2}$. Compound C2 was obtained in a yield of $88 \%(1.0 \mathrm{~g}) .{ }^{1} \mathrm{H}$ NMR $\left(\mathrm{CDCl}_{3}, 300\right.$ $\mathrm{MHz}): \delta 7.70(\mathrm{~d}, 4 \mathrm{H}), 7.28(\mathrm{~d}, 4 \mathrm{H}), 6.66(\mathrm{~s}, 4 \mathrm{H}), 1.65(\mathrm{~s}, 12 \mathrm{H}) \cdot{ }^{13} \mathrm{C}$ NMR (100 MHz, $\left.\mathrm{CDCl}_{3}\right)$ : $\delta$ 147.3, 138.8, 132.9, 131.8, 128.3, 126.7, 121.5, 118.1, 100.8, 26.0.

\section{Synthesis of compound A2}

A Schlenk flask was charged with compound C2 (1.0 mmol), $N$ methyl piperazine $(2.4 \mathrm{mmol})$, sodium tertbutoxide $(2.8 \mathrm{mmol})$, tris(dibenzylideneacetone)dipalladium- $(0) \quad(0.02 \quad \mathrm{mmol}$, $2 \mathrm{~mol} \%$ ), BINAP (0.06 mmol), and toluene (9 $\mathrm{mL})$ under argon. The flask was immersed in an oil bath and heated to $80^{\circ} \mathrm{C}$ with stirring until the starting material had been completely consumed as judged by GC analysis. The mixture was allowed to cool to room temperature, taken up in $\mathrm{CHCl}_{3}(150 \mathrm{~mL})$, filtered, and concentrated. The crude product was then purified further by flash chromatography on silica gel with a mixture of $\mathrm{CHCl}_{3}$ and $\mathrm{CH}_{3} \mathrm{OH}(10: 1, \mathrm{v} / \mathrm{v})$ as eluent to give A2. ${ }^{1} \mathrm{H} \mathrm{NMR}\left(\mathrm{CDCl}_{3}\right.$, $300 \mathrm{MHz}): \delta 7.31(\mathrm{~d}, 4 \mathrm{H}), 7.11(\mathrm{~d}, 4 \mathrm{H}), 6.78(\mathrm{~s}, 4 \mathrm{H}), 3.46(\mathrm{~m}, 8 \mathrm{H})$, $2.87(\mathrm{~m}, 8 \mathrm{H}), 2.56(\mathrm{~s}, 6 \mathrm{H}), 1.64(\mathrm{~s}, 12 \mathrm{H})$.

\section{Synthesis of compound 3}

A flask charged with palladium chloride $(0.16 \mathrm{~g}, 0.9 \mathrm{mmol}), 1,1-$ bis(diphenylphosphino)ferrocene (DPPF) (0.50 g, $0.9 \mathrm{mmol}$ ), potassium acetate $(8.83 \mathrm{~g}, 90 \mathrm{mmol})$, and $4,4,5,5,4,4,5,5$ octamethyl-[2,2]bi[[1,3,2]dioxaborolanyl] (8.38 $\mathrm{g}, 33 \mathrm{mmol})$ was flushed with nitrogen. DMSO $(120 \mathrm{~mL})$ and 1,3,5-tribromobenzene $(3.15 \mathrm{~g}, 10 \mathrm{mmol})$ were then added. After being stirred at $80{ }^{\circ} \mathrm{C}$ for an appropriate period, the product was extracted with benzene, washed with water, and dried over anhydrous magnesium sulfate. Benzene was removed and the residue was purified by column chromatography using silica gel with a mixture of petroleum ether and dichloromethane $(3: 2$, $\mathrm{v} / \mathrm{v}$ ) as eluent to give 3, yield: $87 \%$ (colorless needle crystal). ${ }^{1} \mathrm{H}$ $\mathrm{NMR}\left(\mathrm{CDCl}_{3}, 300 \mathrm{MHz}\right), \delta(\mathrm{ppm}): 1.33(\mathrm{~s}, 36 \mathrm{H}), 8.36(\mathrm{~s}, 3 \mathrm{H}) \cdot{ }^{13} \mathrm{C}$ NMR (100 MHz, $\left.\mathrm{CDCl}_{3}\right): \delta$ 144.1, 83.7, 24.9.

\section{Synthesis of compound B3}

The synthesis of 1,3,5-benzenetriboronic acid was carried out by a modified method of a published procedure. To the solution of pinacol 1,3,5-benzenetriboronic ester $\mathbf{S} 7(1.0 \mathrm{~g}, 2.19 \mathrm{mmol})$ in THF $(24 \mathrm{~mL})$ and water $(6 \mathrm{~mL})$ sodium periodate $(4.22 \mathrm{~g}$, $19.71 \mathrm{mmol}$ ) was added. The cloudy white suspension was stirred overnight at room temperature. Hydrochloric acid (2 M, $0.5 \mathrm{~mL}$ ) was added and the mixture stirred for another 24 hours. 
Methanol (100 mL) was added and the mixture was filtered to remove salts. The filtrate was concentrated by rotator evaporation and hydrochloric acid $(1 \mathrm{M}, 30 \mathrm{~mL})$ was added. The mixture was stirred at room temperature for 2 hours and the resulting precipitate was collected by filtration and dried in vacuum to give triboronic acid compound $\mathbf{B} 3(0.44 \mathrm{~g}, 95 \%)$ as white solid.

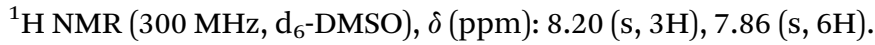
${ }^{13} \mathrm{C}$ NMR (100 MHz, $\left.\mathrm{CD}_{3} \mathrm{OD}\right): \delta(\mathrm{ppm})=142.5$.

\section{Synthesis of iCOFs-A}

Compound A2 (144 mg, $0.214 \mathrm{mmol}$ ) and compound B3 (30 mg, $0.143 \mathrm{mmol}$ ) were loaded into a 1 dram screw-cap vial and suspended in a mixture of mesitylene and dioxane $(1: 1$, $40 \mathrm{~mL}$ ). The dark-blue mixture was sonicated for 15 minutes. Boron trifluoride etherate $(15 \mathrm{~mL}, 0.12 \mathrm{mmol})$ was added, and the mixture was sonicated for another 15 minutes. The dark, heterogeneous mixture was flash frozen in a liquid nitrogen bath. The ampoule neck was flame-sealed in air using a propane torch, which reduced the total length by 20-30 mm. After warming to room temperature the suspension was placed in a gravity convection oven at $120^{\circ} \mathrm{C}$ for six days. The reaction was cooled to room temperature, the ampoule was broken at the scored neck and the mixture was poured onto qualitative filter paper and filtered under vacuum. The resulting dark solid was washed with anhydrous mesitylene and dioxane, and dried in air.

\section{Synthesis of COFs-B}

Compound C2 (144 mg, $0.214 \mathrm{mmol}$ ) and compound B3 (30 mg, $0.143 \mathrm{mmol}$ ) were loaded into a 1 dram screw-cap vial and suspended in a mixture of mesitylene and dioxane $(1: 1$, $40 \mathrm{~mL}$ ). The dark-blue mixture was sonicated for 15 minutes. Boron trifluoride etherate $(15 \mathrm{~mL}, 0.12 \mathrm{mmol})$ was added, and the mixture was sonicated for another 15 minutes. The dark, heterogeneous mixture was flash frozen in a liquid nitrogen bath. The ampoule neck was flame-sealed in air using a propane torch, which reduced the total length by 20-30 mm. After warming to room temperature the suspension was placed in a gravity convection oven at $120^{\circ} \mathrm{C}$ for six days. The reaction was cooled to room temperature, the ampoule was broken at the scored neck and the mixture was poured onto qualitative filter paper and filtered under vacuum. The resulting dark solid was washed with anhydrous $\mathrm{CH}_{3} \mathrm{CN}$ and dried in air.

\section{Results and discussion}

The anthracene-based ionic covalent organic frameworks iCOFs-A was solvothermally synthesized via condensation reaction between $\mathbf{A} 2(0.3 \mathrm{mmol})$ and $\mathbf{B} 3$ (0.2 $\mathrm{mmol})$ (Fig. 1a) in a sealed Pyrex tube using dioxane/mesitylene in the ratio $1: 1$. To demonstrate the effect of ion pairs, we also synthesized the anthracene-based non-ionic covalent organic frameworks COFs-B via condensation reaction between $\mathbf{C 2}(0.3 \mathrm{mmol})$ and B3 (0.2 mmol) (Fig. 1a). Synthetic route of the A2, B3 and C2 was presented in Fig. S1. $\dagger$ Synthetic route of the A2, B3 and C2 was presented in Fig. S1. $\dagger$ In FTIR spectra of iCOFs-A and COFs-B,
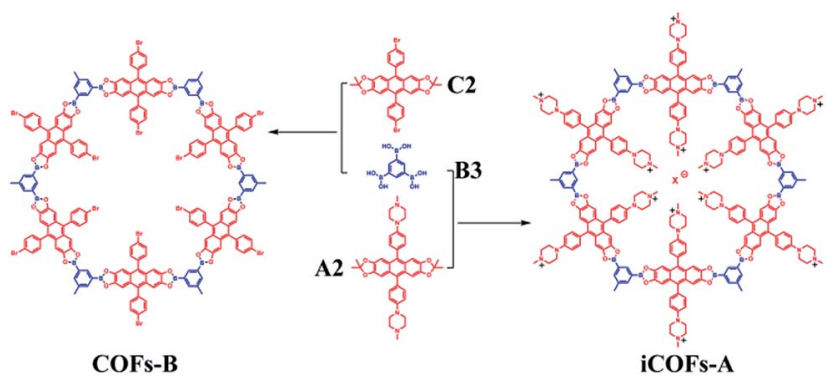

Fig. 1 Synthesis of iCOFs-A and COFs-B

the appearance of the characteristic $\mathrm{B}-\mathrm{O}$ and $\mathrm{C}-\mathrm{O}$ stretching vibrations of the borate ester at $1375 \mathrm{~cm}^{-1}$ and $1231 \mathrm{~cm}^{-1}$ indicated the formation of condensation products (Fig. S2 $\dagger$ ). All the stretching and bending vibrations of the synthesized COFs were summarized in Table S1 and S2. $\dagger$ Moreover, compared ${ }^{13} \mathrm{C}$ CP-MAS solid-state NMR spectra of iCOFs-A and COFs-B with that of $\mathbf{A} 2$ and $\mathbf{C} 2$, a very sharp peak at 20 ppm of the dimethyl group disappeared, which is a strong evidence for the formation of iCOFs-A and COFs-B (Fig. S3†). Since A2 contains $\mathrm{N}$ atoms and $\mathbf{B} 3$ does not, the $\mathrm{C} / \mathrm{N}$ ratio of iCOFs-A can provide the information for the composition and the structure of the $2 \mathrm{D}$ polymer. Elemental analysis showed that the $\mathrm{C} / \mathrm{N}$ ratio of iCOFsA was 10:1 which was consistent with the theoretical value (Fig. S4 $\dagger$ ). In addition, TGA profiles of the iCOFs-A showed that the decomposition temperature was $\sim 237^{\circ} \mathrm{C}$ (Fig. S5 $\dagger$ ).

The PXRD pattern of iCOFs-A displayed two intense peaks at $2 \theta=7.27^{\circ}$ and $7.79^{\circ}$, which correspond to the pore diameters of iCOFs-A (Fig. 2a and c). The broad peaks at $2 \theta=17.6^{\circ}$ and $20.95^{\circ}$ were assigned to the interlayer $\pi-\pi$ stacking distance in iCOFs-A measured to be $\sim 4.3-5.0 \AA$ (Fig. $2 \mathrm{~b}$ and d), which was larger than that $(3.7 \AA)$ of COFs-B (Fig. S6†). This could be attributed to the incorporation of the positively charged nitrogen-atoms, which decreased the $\pi-\pi$ interaction and increased the layer spacing due to electrostatic repulsion. This result provides a positive feedback that it may be possible to achieve self-exfoliation of iCOFs in solution. Inspired by this result, we have successfully prepared ionic covalent organic nanosheets iCONs-A by selfexfoliation of iCOFs-A in polar solvents. (a)

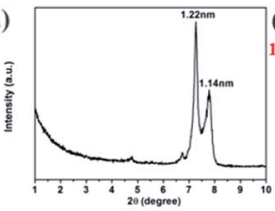

(b)

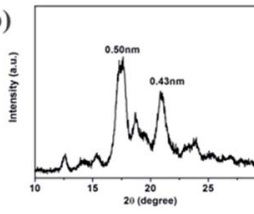

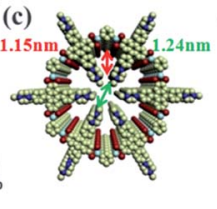

(d)

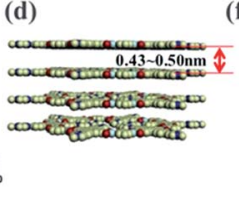

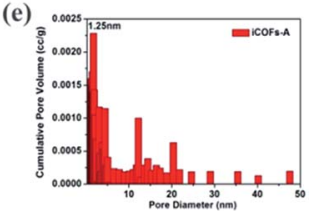

(f)

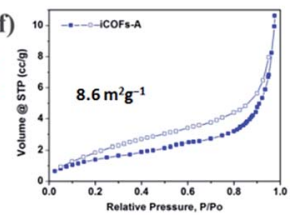

Fig. 2 (a, b) Experimental PXRD patterns of iCOFs-A. (c, d). Simulated PXRD pattern of iCOFs-A in eclipsed mode. (e) Pore size distribution of iCOFs-A. (f) Nitrogen sorption isotherms for iCOFs-A at $77 \mathrm{~K}$. 
Permanent porosity of iCOFs-A and COFs-B were verified by $\mathrm{N}_{2}$ adsorption isotherms of the activated samples at $77 \mathrm{~K}$, the results indicated that both of them followed type-II reversible adsorption isotherm. The Brunauer-Emmett-Teller (BET) surface area of iCOFs-A and COFs-B were calculated to be $8.6 \mathrm{~m}^{2} \mathrm{~g}^{-1}$ (Fig. 2f) and $16 \mathrm{~m}^{2} \mathrm{~g}^{-1}$ (Fig. S7†), respectively. The lower surface area of iCOFs-A could be ascribed to poor layer stacking, small pore diameter, and pore blocking by the counter anions. ${ }^{42}$ Pore size distribution was calculated on the basis of nonlocal density function theory (NLDFT). The pore diameters of iCOFs-A and COFs-B were calculated to be $1.25 \mathrm{~nm}$ (Fig. 2e) and $1.71 \mathrm{~nm}$ (Fig. S7†). Due to the lack of proper channelled pore structure and the pore blocking by counter anions, the size distribution of iCOFs-A was not as sharp as that in COFs-B.

It is well known that ionic organic molecules prefer to dissolve in polar solvents rather than nonpolar solvents due to the difference in solvation ability of the ionic molecules in the solvents. To explore self-exfoliation of iCOFs-A in solution, we performed dialysis of iCOFs-A in organic solvents with different polarities.

The effect of solvent polarity on the self-exfoliation of the iCOFs-A was tracked by a combination of scanning electron microscopy (SEM), transmission electron microscopy (TEM), atomic force microscopy (AFM) and light-scattering (Fig. 3). First, iCOFs-A was dispersed in dioxane/mesitylene (1/1) and characterized by electron microscopy. TEM images (Fig. 3a) and SEM images (Fig. 3d) showed that iCOFs-A possessed a layer-bylayer structure with an average diameter of $3.5 \pm 0.5 \mu \mathrm{m}$, which consistent with the results of dynamic light scattering (Fig. 3g). Then, the iCOFs-A dispersed in dioxane/mesitylene was dialyzed respectively in dioxane and acetonitrile through a dialysis membrane with a molecular weight cut-off (MWCO) of 1000. Interestingly, the results revealed that hollow nanospheres were formed in dioxane with an average diameter of $250 \pm 50 \mathrm{~nm}$ (Fig. 3b, e and h) and an average thickness of $7.5 \pm 0.5 \mathrm{~nm}$ (Fig. S8 $\dagger$ ), whereas nanosheets were obtained in acetonitrile

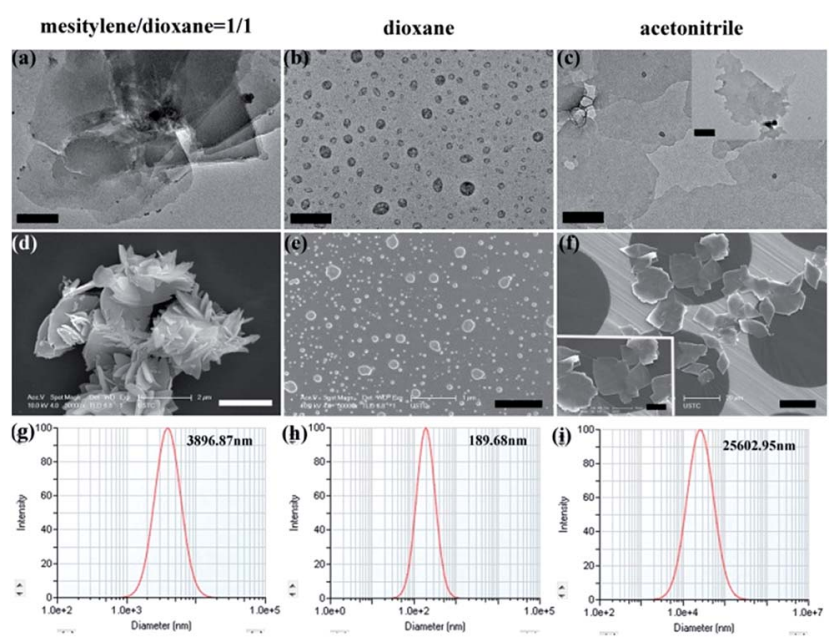

Fig. 3 Monitoring the morphological transformation from iCOFs-A to iCONs-A by TEM (a-c), SEM ( $d-f)$ and DLS studies ( $g-i)$. Scale bars: $(a)$ $0.5 \mu \mathrm{m}$, (b) $0.5 \mu \mathrm{m}$, (c) $0.2 \mu \mathrm{m}$ (inlet image: $2 \mu \mathrm{m}$ ), (d) $2 \mu \mathrm{m}$, (e) $1 \mu \mathrm{m}$, (f) $20 \mu \mathrm{m}$ (inlet image: $10 \mu \mathrm{m}$ ). with an average diameter of $20 \pm 5 \mu \mathrm{m}$ (Fig. 3c, $\mathrm{f}$ and i) and an average thickness of $3.5 \pm 0.5 \mathrm{~nm}$ (Fig. S8 $\dagger$ ). According to the recent reports, ${ }^{\mathbf{4 3 - 4 5}}$ polymers tend to form nanocapsules in poor solvents, whereas they prefer forming $2 \mathrm{D}$ films in good solvents. In our case (Fig. 4), since the mixture of dioxane/mesitylene (1/ 1) with low solvation power is not a good solvent for iCOFs-A, the multilayered iCOFs-A was obtained. When dioxane/ mesitylene was replaced by dioxane, solvation of the iCOFs-A with dioxane led to a decrease in the interaction of two layers and an increase in the layer spacing, and finally, the bulk iCOFsA were exfoliated into thin iCOFs-A sheets with a few layers or even a monolayer. However, the thin iCOFs-A sheets tend to curl into a ball to reduce the Gibbs-energy, ${ }^{43}$ this can be attributed to the solvation power and polarity of dioxane, both of which are not large enough to stabilize the peeled layers. Compared with dioxane, acetonitrile possesses stronger solvation ability, so not only the bulk iCOFs-A can be exfoliated by acetonitrile, but also the stable few layered iCONs-A sheets can be obtained in acetonitrile.

The self-exfoliation experiments of iCOFs-A were performed in various organic solvents, but only a few of organic solvents with relatively strong polarity are effective for the self-exfoliation of iCOFs-A, including DMF, DMSO, $\mathrm{CH}_{3} \mathrm{CN}$, and $\mathrm{CH}_{3} \mathrm{OH}$ (Fig. S11†). The weight and volume ratio between iCOFs-A and organic solvent for the self-exfoliation was $0.5 \mathrm{mg} \mathrm{mL} \mathrm{m}^{-1}$. As a control experiment, we also performed dialysis of the COFs-B in solvents with different polarities, the results demonstrated that no change in morphology was observed (Fig. S9†), indicating no selfexfoliation occurred. Obviously, both electrostatic repulsion and solvation are important for the self-exfoliation of iCOFs-A. First, the electrostatic repulsion led to the weaker $\pi-\pi$ interaction between the layers and the larger layer spacing, which are favorable for the intercalating of solvent. Then, the solvation of polar solvents with iCOFs-A resulted in their self-exfoliation. Therefore, the incorporation of ionic groups is of great significance for self-exfoliation of the COFs in solution.

Since few-layered nanosheets of the iCONs-A can easily be obtained from self-exfoliation of the iCOFs-A in polar solvents, we have successfully prepared uniform, free-standing CON thin films of iCONs-A (Fig. S10a and $b \dagger$ ), indicating a significant impact on processing and applications of COFs. Moreover, we

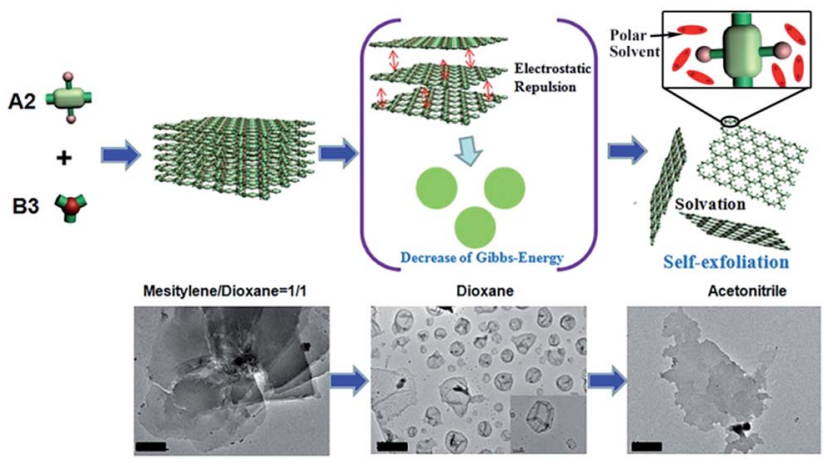

Fig. 4 Schematic illustration of the formation of 2D ionic covalent organic nanosheets. Scale bars: $0.5 \mu \mathrm{m}$ (left), $0.5 \mu \mathrm{m}$ (middle), $2 \mu \mathrm{m}$ (right). 
investigated the electrical conductivity and photoconductivity of the self-exfoliated nanosheets by casting a thin film of iCONs-A on $\mathrm{SiO}_{2}$ wafer. The micro-gap electrodes were fabricated by photolithography silicon wafer covered with a $300 \mathrm{~nm}$ thick $\mathrm{SiO}_{2}$ dielectric layer. The gold electrode pair was $50 \mu \mathrm{m}$ long and $5 \mu \mathrm{m}$ wide and the film thickness was about $50 \mu \mathrm{m}$. Based on the obtained data, smaller current was observed with iCONs-A ${ }^{\mathbf{4 6}}$ which may be ascribed to the weaker stacking (Fig. S10d $\dagger$ ). Then, on irradiation with visible light from a xenon lamp, the characteristics curve showed larger current, which illustrated the photoresponse of the film. In order to investigate the photocurrent response, we measured the transient photocurrent of the device at a bias voltage of $2 \mathrm{~V}$ (Fig. S10c $\dagger$ ). The photosensitivity was found to be 3.86. Transient photocurrents were steady and reproducible during on-off cycles of the visible light irradiation. The photoresponse of iCONs-A was likely due to their $\pi-\pi$ overlap among re-accumulated nanosheets (Fig. S10d $\dagger$ ). ${ }^{47}$ We believe that it is possible to obtain nanosheets of iCOFs with excellent electroconductive and photoconductive properties by rationally designing composition and structure of the iCONs.

\section{Conclusions}

In conclusion, both the anthracene-based ionic covalent organic frameworks iCOFs-A and non-ionic covalent organic frameworks COFs-B were designed and synthesized and their self-exfoliation was investigated in different organic solvents. It was demonstrated that iCOFs-A possesses self-exfoliating property in organic solvents with high polarity, but COFs-B does not. The results indicated that the incorporation of ionic moiety into COFs is of great significance for self-exfoliation of the COFs in solution. Based on electrostatic repulsion and solvation, ionic covalent organic nanosheets iCONs-A can be obtained from self-exfoliation of iCOFs-A in organic solvents with high polarity. Moreover, few layered iCONs-A was used to prepare uniform thin films on $\mathrm{SiO}_{2}$, which exhibited explicit electroconductive and photoconductive properties. Therefore, this work not only provided a new approach for self-exfoliation of boron-based COFs, but also opened a new perspective for processing and applications of COFs materials.

\section{Conflicts of interest}

There are no conflicts to declare.

\section{Acknowledgements}

The authors are grateful for financial support from the National Natural Science Foundation of China (No. 21674101).

\section{Notes and references}

1 K. S. Novoselov, A. K. Geim, S. V. Morozov, D. Jiang, Y. Zhang, S. V. Dubonos, I. V. Grigorieva and A. A. Firsov, Science, 2004, 306, 666-669.

2 J. Sakamoto, J. V. Heijst, O. Lukin and A. D. Schlüter, Angew. Chem., Int. Ed., 2009, 48, 1030-1069.
3 P. Kissel, R. Erni, W. B. Schweizer, M. D. Rossell, B. T. King, T. Bauer, S. Götzinger, A. D. Schlüter and J. Sakamoto, Nat. Chem., 2012, 4, 287-291.

4 R. Bhola, P. Payamyar, D. J. Murray, B. Kumar, A. J. Teator, M. U. Schmidt, S. M. Hammer, A. Saha, J. Sakamoto, A. D. Schlüter and B. T. King, J. Am. Chem. Soc., 2013, 135, 14134-14141.

5 P. Kissel, D. J. Murray, W. J. Wulftange, V. J. Catalano and B. T. King, Nat. Chem., 2014, 6, 774-778.

6 M. J. Kory, M. Wörle, T. Weber, P. Payamyar, S. W. van de Poll, J. Dshemuchadse, N. Trapp and A. D. Schlüter, Nat. Chem., 2014, 6, 779-784.

7 X. D. Zhuang, Y. Y. Mai, D. Q. Wu, F. Zhang and X. L. Feng, Adv. Mater., 2015, 27, 403-427.

8 S. L. Cai, W. G. Zhang, R. N. Zuckermann, Z. T. Li, X. Zhao and Y. Liu, Adv. Mater., 2015, 27, 5762-5770.

9 W. Bai, Z. W. Jiang, A. E. Ribbe and S. Thayumanavan, Angew. Chem., Int. Ed., 2016, 55, 10707-10711.

10 A. P. Côté, A. I. Benin, N. W. Ockwig, A. J. Matzger, M. O'Keeffe and O. M. Yaghi, Science, 2005, 310, 1166-1170.

11 H. M. El-Kaderi, J. R. Hunt, J. L. Mendoza-Cortes, A. P. CÔté, R. E. Taylor, M. O'Keeffe and O. M. Yaghi, Science, 2007, 316, 268-272.

12 A. P. CÔté, H. M. El-Kaderi, H. Furukawa, J. R. Hunt and O. M. Yaghi, J. Am. Chem. Soc., 2007, 129, 12914-12915.

13 R. W. Tilford, S. J. Mugavero III, P. J. Pellechia and J. J. Lavigne, Adv. Mater., 2008, 20, 2741-2746.

14 S. Wan, J. Guo, J. Kim, H. Ihee and D. Jiang, Angew. Chem., Int. Ed., 2008, 47, 8826-8830.

15 S. Wan, J. Guo, J. Kim, H. Ihee and D. Jiang, Angew. Chem., Int. Ed., 2009, 48, 5439-5442.

16 E. L. Spitler and W. R. Dichtel, Nat. Chem., 2010, 2, 672-677. 17 A. Nagai, Z. Guo, X. Feng, S. Jin, X. Chen, X. Ding and D. Jiang, Nat. Commun., 2011, 2, 536-543.

18 D. N. Bunck and W. R. Dichtel, Angew. Chem., Int. Ed., 2012, 51, 1885-1889.

19 P. Kuhn, M. Antonietti and A. Thomas, Angew. Chem., Int. Ed., 2008, 47, 3450-3453.

20 R. Palkovits, M. Antonietti, P. Kuhn, A. Thomas and F. Schüth, Angew. Chem., Int. Ed., 2009, 48, 6909-6912.

21 M. J. Bojdys, J. Jeromenok, A. Thomas and M. Antonietti, Adv. Mater., 2010, 22, 2202-2205.

22 C. E. Chan-Thaw, A. Villa, P. Katekomol, D. Su, A. Thomas and L. Prati, Nano Lett., 2010, 10, 537-541.

23 C. E. Chan-Thaw, A. Villa, L. Prati and A. Thomas, Chem.Eur. J., 2011, 17, 1052-1057.

24 F. J. Uribe-Romo, J. R. Hunt, H. Furukawa, C. Klock, M. O'Keeffe and O. M. Yaghi, J. Am. Chem. Soc., 2009, 131, 4570-4571.

25 F. J. Uribe-Romo, C. J. Doonan, H. Furukawa, K. Oisaki and O. M. Yaghi, J. Am. Chem. Soc., 2011, 133, 11478-11481.

26 S.-Y. Ding, J. Gao, Q. Wang, Y. Zhang, W.-G. Song, C.-Y. Su and W. Wang, J. Am. Chem. Soc., 2011, 133, 19816-19822.

27 S. Wan, F. Gandara, A. Asano, H. Furukawa, A. Saeki, S. K. Dey, L. Liao, M. W. Ambrogio, Y. Y. Botros, X. Duan, S. Seki, J. F. Stoddart and O. M. Yaghi, Chem. Mater., 2011, 23, 4094-4097. 
28 S. Wan, J. Guo, J. Kim, H. Ihee and D. L. Jiang, Angew. Chem., Int. Ed., 2008, 47, 8826-8830.

29 S. Wan, F. Gandara, A. Asano, H. Furukawa, A. Saeki, S. K. Dey, L. Liao, M. W. Ambrogio, Y. Y. Botros, X. F. Duan, S. Seki, J. F. Stoddart and O. M. Yaghi, Chem. Mater., 2011, 23, 4094-4097.

30 X. S. Ding, J. Guo, X. Feng, Y. Honsho, J. D. Guo, S. Seki, P. Maitarad, A. Saeki, S. Nagase and D. L. Jiang, Angew. Chem., Int. Ed., 2011, 50, 1289-1293.

31 X. S. Ding, L. Chen, Y. Honsho, X. Feng, O. Saengsawang, J. D. Guo, A. Saeki, S. Seki, S. Irle, S. Nagase, V. Parasuk and D. L. Jiang, J. Am. Chem. Soc., 2011, 133, 14510-14513.

32 X. Feng, L. L. Liu, Y. Honsho, A. Saeki, S. Seki, S. Irle, Y. Dong, A. Nagai and D. L. Jiang, Angew. Chem., 2012, 124, 2672-2676.

33 I. Berlanga, M. L. Ruiz-Gonzalez, J. M. Gonzalez-Calbet, J. L. G. Fierro, R. Mas-Balleste and F. Zamora, Small, 2011, 7, 1207-1211.

34 S. Chandra, S. Kandambeth, B. P. Biswal, B. Lukose, S. M. Kunjir, M. Chaudhary, R. Babarao, T. Heine and R. Banerjee, J. Am. Chem. Soc., 2013, 135, 17853-17861.

35 D. N. Bunck and W. R. Dichtel, J. Am. Chem. Soc., 2013, 135, 14952-14955.

36 M. A. Khayum, S. Kandambeth, S. Mitra, S. B. Nair, A. Das, S. S. Nagane, R. Mukherjee and R. Banerjee, Angew. Chem., Int. Ed., 2016, 55, 15604-15608.
37 S. Mitra, S. Kandambeth, B. P. Biswal, A. Khayum, C. K. Choudhury, M. Mehta, G. Kaur, S. Banerjee, A. Prabhune, S. Verma, S. Roy, U. K. Kharul and R. Banerjee, J. Am. Chem. Soc., 2016, 138, 2823-2828.

38 Y. Li and R. T. Yang, AIChE J., 2008, 54, 269-279.

39 L. M. Lanni, R. W. Tilford, M. Bharathy and J. J. Lavigne, J. Am. Chem. Soc., 2011, 133, 13975-13983.

40 E. L. Spitler and W. R. Dichtel, Nat. Chem., 2010, 2, 672-677. 41 E. L. Spitler, M. R. Giovino, S. L. White and W. R. Dichtel, Chem. Sci., 2011, 2, 1588-1593.

42 H. Ma, B. L. Liu, B. Li, L. M. Zhang, Y. G. Li, H. Q. Tan, H. Y. Zang and G. S. Zhu, J. Am. Chem. Soc., 2016, 138, 5897-5903.

43 K. Baek, I. Hwang, I. Roy, D. Shetty and K. Kim, Acc. Chem. Res., 2015, 48, 2221-2229.

44 J. Kim, K. Baek, D. Shetty, N. Selvapalam, G. Yun, N. H. Kim, Y. H. Ko, K. M. Park, I. Hwang and K. Kim, Angew. Chem., Int. Ed., 2015, 54, 2693-2697.

45 R. K. Shukla and K. K. Raina, Int. J. Mod. Phys. B, 2009, 23, 5075-5083.

46 S. Wan, J. Guo, J. Kim, H. Ihee and D. L. Jiang, Angew. Chem., Int. Ed., 2009, 48, 5439-5442.

47 X. J. Ma, Y. B. Zhang, Y. F. Zhang, C. Peng, Y. Che and J. C. Zhao, Adv. Mater., 2015, 27, 7746-7751. 